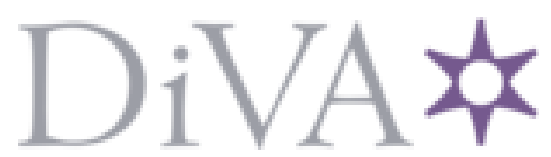

http://www.diva-portal.org

\title{
Postprint
}

This is the accepted version of a paper presented at CHI 2016, ACM International Conference on Human-Computer Interaction, May 7 - 12 at San Jose, CA, USA..

Citation for the original published paper:

Handberg, L., Gullström, C., Kort, J., Nyström, J. (2016)

SharedSpaces Mingle.

In: Proceedings of the 34th annual ACM conference on Human factors in computing systems (CHI

'16), ACM, May 2016. ACM Digital Library

Extended abstracts; ACM Digital Library.

N.B. When citing this work, cite the original published paper.

(C) Handberg, Gullström, Kort, Nyström $\mid$ ACM 2016. This is the author's version of the work. It is posted here for your personal use. Not for redistribution. The definitive Version of Record was published in Proceedings of the 34th annual ACM conference on Human factors in computing systems CHI '16, http://dx.doi.org/10.1145/2851581.2889469.

Permanent link to this version:

http://urn.kb.se/resolve?urn=urn:nbn:se:kth:diva- 185584 


\section{Images below:}

Screenshots from the SharedSpaces video figure

with additional

explanatory images

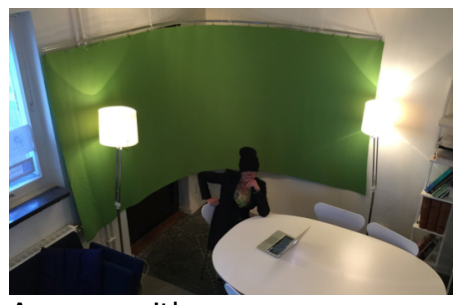

A room with a green screen and participant 1 .

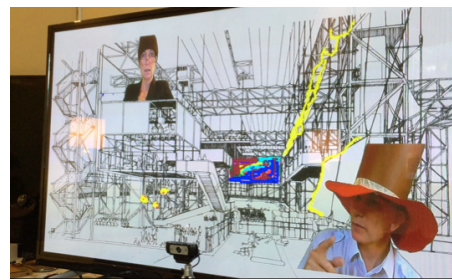

Shot of screen with two

participants in SharedSpaces.

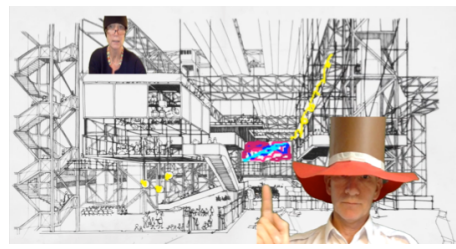

Screenshot from the same screen.

\section{SharedSpaces Mingle}

\author{
Leif Handberg \\ Charlie Gullström \\ KTH \\ Stockholm. \\ leifh@kth.se \\ charlieg@kth.se \\ Joke Kort \\ TNO \\ Groningen \\ joke.kort@tno.nl \\ Jimmy Nyström \\ LTU \\ Luleå \\ jimmy.nystrom@ltu.se
}

Permission to make digital or hard copies of part or all of this work for personal or classroom use is granted without fee provided that copies are not made or distributed for profit or commercial advantage and that copies bear this notice and the full citation on the first page. Copyrights for thirdparty components of this work must be honored. For all other uses, contact the Owner/Author.

Copyright is held by the owner/author(s). CHI'16 Extended Abstracts, May 07-12, 2016, San Jose, CA, USA ACM 978-1-4503-4082-3/16/05. http://dx.doi.org/10.1145/2851581.2889469

\begin{abstract}
SharedSpaces is a WebRTC design prototype that creates a virtual media space where people can mingle and interact. Although you are in different locations, you appear side by side in front of a chosen backdrop. This interactive installation addresses spatial and social connectedness, stressing the importance of integrating architectural and spatial features to support complex social dynamics in mediated interaction. The tool engages users in manipulating their real-time videostreams, creatively co-designing a shared mediated space that fits a contextual need. It supports social dynamics by allowing users to draw and paint together and to move and resize video streams. Further, it enhances grounding and social cues by merging videostreams and space, representing users as if they were in the same space. Standard and easily available equipment is used. Recent user studies show that a seamless integration of space, social dynamics and shared activity benefits the experience of presence, naturalness, immersion/engagement and social
\end{abstract} connectedness.

\section{Author Keywords}

Naturalness; immersion; social, spatial presence; design; spatial, social connectedness, WebRTC; media, mediated space; audio/video communication; architecture. 


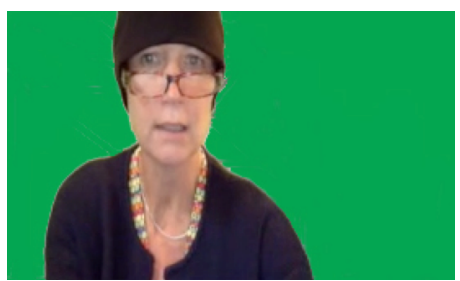

Camera view of participant 1 without green screen background removal.

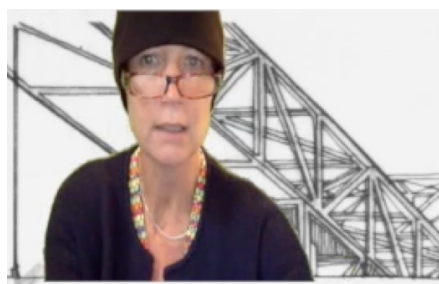

Camera view of participant 1 with green screen background removal.

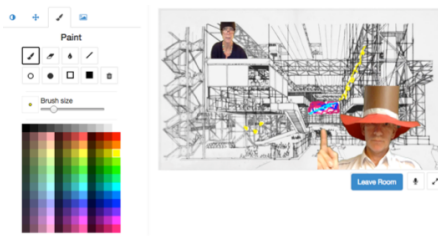

Drawing possible with the drawing tool.

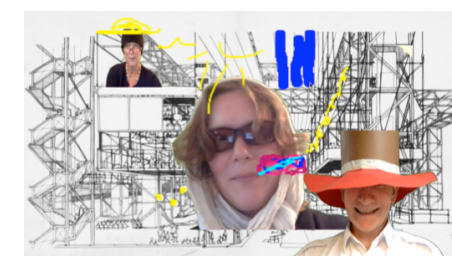

Third person enters.

\section{ACM Classification Keywords}

H.5.m. Information interfaces and presentation (e.g., $\mathrm{HCI}$ ): Miscellaneous.

\section{Introduction}

In the last decade there has been numerous attempts to reach beyond the "talking heads" and the 16:9 paradigm [8]. The most elaborate solutions have integrated heavy hardware in spaces where users benefit from how activity and social space are brought together through design, yet in very costly interiors

[3]. On the other hand, new standards for web-based communication (WebRTC, HTML5) facilitate a

representation of participants and activity space, simply via a web interface, with the help of a webcam and computer. In terms of further development, many web applications for mediated interaction focus on technical maturation (e.g. automatic bandwidth reduction, switching microphone on/off through sound detection algorithms, adding additional tools for cooperative tasks, etc.). In contrast, we hypothesize that multiparty interaction, and in particular naturalness [7], and immersion/engagement, will benefit from spatial, social and shared activity features in one seamless (virtual) space. Our ongoing design research specifically addresses the fusion of spatial connectedness (a term describing how well users perceive that real space is integrated with virtual space) and social connectedness (how well users perceive social dynamics/cues and their relationship with others in mediated space during interaction).

In the larger context our research seeks to create a seamless mediated space for social interaction and shared activity by integrating live and/or stored media streams combined with software applications (e.g. physical sensors and actuators) that can provide live feedback and empower users to actively control features in local (as well as remote) and virtual spaces.

\section{Our work in context}

Over twenty-five years have passed since Bill Buxton and his colleagues [1] used architectural spaces as a reference for the Hydra studies in which they showed the importance of spatial cues and design as a prerequisite for presence in shared 'person spaces' and remote collaboration. They challenged media, as a new field, to achieve what architecture as a mature discipline already does: support physical proximity by activity-based design.

Today, the field of presence is arguably mature nevertheless still lacks in adequate terminology to also discuss how spatial connectedness and the experience of proximity and activity benefit and support the social connectedness. Spatial connectedness indicates that architectural design is as important in mediated environments as in real spaces for human interaction. In architecture, spatial features such as proximity, visibility and layout may stimulate interaction and collaboration [5]. Architectural design equally creates spatial dividers, walls and barriers by e.g. geographically separating offices or domestic environments, seeking to harbor privacy, security, but also (social) exclusion and loneliness [2]. Equally, in shared mediated spaces, the individual experience of presence is negotiated as part of a process to establish trust and confirmation from the remote party/parties $[4,6]$. An ongoing construction of a sense of place is informed by a series of spatial and social cues, i.e.

'reality tests' carried out by participants, who seek fast, consistent and reliable confirmation that the interaction 


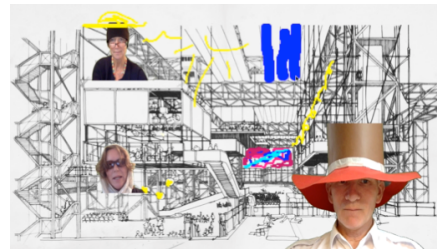

Third person scaled and moved with video control tool.

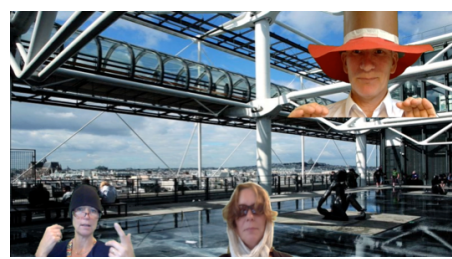

Change of background.

Backgrounds can be chosen from a predifined set of images, or be uploaded from any of the participants computers. The background can also be a live or recorded video feed. The background can be altered to support the communication context and to help a conversation move between formal and informal stages.

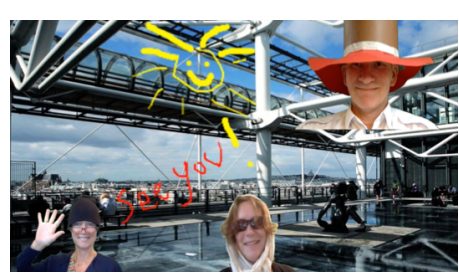

can be trusted - in which case they can temporarily agree to suspend their judgment in favor of a 'willing suspension of disbelief'. In a broader context, social connectedness is a layered construct that describes how people relate and connect with one another in society and social groups [9]. The linking factor between spatial and social connectedness is the ambition to design mediated spaces that support and enhance trust-building processes and secure this common ground and interaction rituals.

In sum, the most important prerequisites for spatial and social connectedness are: a sense of shared space is established (presence); and to the extent that necessary cues needed for social interaction, grounding, and common understanding are secured (social presence); as well as a sense of naturalness in relation to the environment as a whole and to the individual self (naturalness); and an integration of activities (interaction rituals) that express social dynamics and create involvement (immersion \& engagement).

\section{Aim of the work}

Our aim with this interactive installation is to present how our design prototype SharedSpaces serves the seamless merging of spaces, social interaction and shared activities in a virtual space, hereby seeking to enhance the experience of (social) presence, naturalness, immersion and engagement and social connectedness.

\section{SharedSpaces design}

SharedSpaces adds to the quality of experience of spatial and social connectedness by representing multiple users side by side on a screen, with the possibility to draw together and to customize their sizes and backdrops. This engages users in manipulating their real-time video-streams, creatively co-designing a shared mediated space (media space) that fits their contextual needs. It supports social dynamics by allowing users to change position and resize video streams and enhances grounding and social cues by merging video-streams and space, representing users as if they were "side by side".

Video-streams are merged using a WebRTC greenscreen component for background removal. Users can easily upload new backdrops, images or live-feeds. Since the manipulation of video-streams is so easy, a user could also host others in his/her real space by simply removing the green background in his/her physical space or adding an additional video-stream of his/her space and maximizing this video-stream using it as a backdrop. Others will be merged into this backdrop. Furthermore, users can draw and paint on the screen/space as a whole, creating possibilities for co-creation and strengthening social exchange.

All that users need in order to try this prototype is a computer with webcam, an Internet connection, Google Chrome (or another browser supporting WebRTC), and a green background. No additional software needs to be installed. Users will be asked to login. The url to the prototype is https://r1.kth.se/smartspaces/fun.

\section{A description of the audience the work intends to serve.}

By offering a context of social mingling, the interactive installation seeks to attract conference participants, such as interaction designers, experience designers, architects, WebRTC developers and IoT service 
innovators. From previous exposure and from user studies we have noticed that the prototype is attractive to a wide range of target audiences, e.g. teenagers.

\section{Relevance of the work to the CHI community}

SharedSpaces offers a fun, novel and aesthetically appealing approach by engaging users in multiple locations to manipulate their real-time video-streams, hereby targeting co-creation from the integration of spatial and social features. The design prototype SharedSpaces adds a spatial quality of experience by representing the users side by side in a shared virtual space. It is an example of innovative services increasingly available, inviting users to seamlessly move between real and virtual spaces using a range of previously separated media channels. This provides new challenges for $\mathrm{HCI}$ designers and calls for interdisciplinary research, new conceptual tools and methodologies to adequately design and represent content in ways that are meaningful and contribute quality of experience to users in various contexts. Recent user studies show that a seamless integration of space, social dynamics and shared activity benefits the experience of presence, naturalness, immersion/ engagement and social connectedness. Continued research seeks to enhance the current prototype and to carry out evaluations in various contexts, in order to further explore the relationship between spatial and social connectedness in the context of shared activities in different domains (work, domestic and public environment).

\section{Acknowledgements}

This work was performed within the EU FP7 COMPEIT project (www.compeit.eu). The COMPEIT project explores the future of connected media and creates a web-based system for highly interactive, personalized, shared media experiences. The project is funded by the European Union.

\section{References}

1. Bill W. Buxton. 1992. Telepresence: integrating shared task and person spaces. In the Proceedings of Graphics Interface '92, 123-129.

2. John T. Cacioppo and William Patrick. Loneliness: Human Nature and the Need for Social Connection. 2009. W. W. Norton \& Company.

3. Cisco Immersive Telepresence. Rerieved September 24, 2015 from http://www.ivci.com/products/cisco/ciscoimmersive-telepresence/

4. Gullström, C. 2012. Design Frictions. In Springer AI \& Society Journal of Knowledge, Culture and Communication, Special issue Witnessed Presence.

5. Hillier, B. 1996. Space is the Machine: A Configurational Theory of Architecture (Cambridge: Cambridge University Press)

6. IJsselsteijn, W.A. (2004). Presence in depth. Doctoral dissertation. Eindhoven: Eindhoven University of ITechnology

7. Ned Kock. 2002. Evolution and Naturalness: A look at E-communication through a Darwinian Theoretical Lens. In Proceedings of the TwentyThird International Conference on Information Systems (ICIS'02).

8. Joke Kort, Charlie Gullstrom, Harold C. Nefs. 2013. Beyond talking heads - Towards sharing life. Proceedings of the CHI'13 workshop The Future of Personal Video Communication: Moving Beyond Talking Heads to Shared Experiences.

9. Sabine Wildevuur, Dick van Dijk, Anne Äyväri, Mie Bjerre, Thomas Hammer-Jakobsen, and Jesper Lund. 2013. Connect - Design for an Emphatic Society. BIS Publishers. 